$67-68$

\title{
Development of a whole disc organ culture system to study human intervertebral disc
}

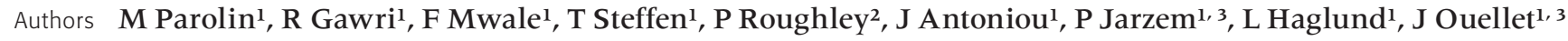
Institutions $\quad{ }^{1}$ Department Of Surgery, McGill University, Montreal, Quebec, Canada

${ }^{2}$ Shriner's Hospital for Children, McGill University, Montreal, Quebec, Canada

${ }^{3}$ McGill Scoliosis and Spine Center, McGill University, Montreal, Quebec, Canada

\section{ABSTRACT}

Study type: Basic science

Objective: Low back pain is one of the most common health problems [1] and is strongly associated with intervertebral disc degeneration, (IVD). Current treatments remove the symptoms without reversing or even retarding the underlying problem. Development of new therapy for the regeneration of the degenerative IVD is complicated by the lack of a validated long-term organ culture model in which therapeutic candidates can be studied. The object of this study was to develop, optimize, and validate an organ culture model for human IVD, allowing for the study of degeneration and the potential for regeneration of the human IVD.

Methods: From eleven donors, an average of 5-6 IVDs were obtained. Inclusion criteria were; age between 50 and 70 years old, no history of cancer, chemotherapy, diabetes, or liver cirrhosis. An x-ray of the harvested spine was done to assess the grade of degeneration. Three different methods for isolating the discs were studied: with bony endplate (BEP), without endplate (NEP), and with cartilage endplate (CEP). Discs were cultured for 4 weeks without external load, in Dulbecco's modified eagle media with glucose and fetal bovine serum (FBS). Four different combinations of concentrations of glucose and FBS were compared: low glucose-low FBS, low glucose-high FBS, high glucose-low FBS, and high glucose-high FBS [2]. Shortterm cultures ( 1 week) were performed to compare the cell viability of the three methods of isolating the discs. Swelling potential on NEP and CEP discs from the same donor were evaluated. After four weeks of culture, a $4 \mathrm{~mm}$ punch was taken from CEP discs and cell viability was evaluated using a live/dead assay with confocal microscopy.

This research has received funding from CIHR, AOSpine Foundation, and Shriners of North America 
Results: Analyzing the potential of swelling in CEP discs, there was an increase in volume to a maximum of $25 \%$ and retention of shape and morphology. Whereas in NEP discs, there was an excessive deformation and a two-fold time increase in volume than CEP discs. The cell viability in short-term cultures is around $40 \%-50 \%$ in the BEP model, 50\%-60\% in the NEP model and $>96 \%$ in the CEP model. BEP isolated discs show endplate necrosis that begins after 4 days of culture. Cell viability in CEP discs was evaluated at 4 weeks in three different areas of the disc: nucleus pulposus, inner annulus fibrosus, and outer annulus fibrosus. We found no difference in live cells (>96\%) between the four different concentrations of FBS and glucose (Table 1).

Conclusions: We have developed a novel method to isolate human IVDs and optimized the culture conditions. The CEP method has been proven to be superior to the previous models (NEP and BEP) in cell viability and maintaining physiologic swelling [3]. In the longterm cultures, the CEP system maintained sufficient nutrient supply and high cell survival in all regions of the discs even with low concentrations of FBS and glucose. The availability of an intact disc organ culture system has a considerable advantage over the culture of isolated disc cells, as it maintains the cells in their unique microenvironment, making any response to catabolic or anabolic agents more physiologically relevant.

\begin{tabular}{lcll}
\hline $\begin{array}{l}\text { Table } 1 \\
\text { under different glucose and FBS* }\end{array}$ & \multicolumn{3}{c}{ cell visibility after 4 weeks of organ culture } \\
un (\%) & $\begin{array}{l}\text { iAF (\%) } \\
\text { viability }\end{array}$ & $\begin{array}{l}\text { oAF (\%) } \\
\text { viability }\end{array}$ \\
\hline vulture Condition & $98.44 \pm 1.60$ & $97.42 \pm 2.75$ & $98.06 \pm 3.58$ \\
\hline HIGH GLUCOSE + HIGH FBS & & & \\
\hline HIGH GLUCOSE + LOW FBS & $97.61 \pm 1.58$ & $97.37 \pm 0.93$ & $96.05 \pm 2.75$ \\
\hline LOW GLUCOSE + LOW FBS & $97.18 \pm 1.22$ & $97.81 \pm 0.68$ & $96.23 \pm 2.51$ \\
\hline LOW GLUCOSE + HIGH FBS & $96.38 \pm 2.48$ & $97.10 \pm 1.85$ & $97.19 \pm 1.95$ \\
\hline
\end{tabular}

Cell visibility after four weeks of organ culture under different glucose and FBS concentrations

$*$ FBS $=$ Fetal bovine serum

\section{REFERENCES:}

1. Andersson GB (1998) Epidemiology of low back pain. Acta Orthop Scand Suppl. Jun; 281:28-31.

2. Johnson WE, Stephan S, Roberts S (2008) The influence of serum, glucose and oxygen on intervertebral disc cell growth in vitro: implications for degenerative disc disease. Arthritis Res Ther; $10(2)$ :R46

3. Roberts N, Hogg D, Whitehouse GH, et al (1998) Quantitative analysis of diurnal variation in volume and water content of lumbar intervertebral discs. Clin. Anat; 11(1):1-8. 